\title{
Meta-analysis comparing "freeze-all strategy" with traditional cycle of in vitro fertilization and fresh embryo transfer
}

\author{
Diego Mendes Do Carmo1*, Eduardo Camelo de Castro ${ }^{1,2}$, Amanda Rincon Godinho1, \\ Antônio Márcio Teodoro Cordeiro Silva ${ }^{1}$ \\ 1Pontifícia Universidade Católica de Goiás (PUC Goiás), Goiânia, Goiás, Brasil \\ ${ }^{2}$ Humana Medicina Reprodutiva, Goiânia, Goiás, Brasil
}

\begin{abstract}
Objective: To perform a meta-analysis comparing the outcomes of the freeze-all strategy with traditional cycles of in vitro fertilization and fresh embryo transfer. Methods: We used the Pubmed, EMBASE and Cochrane virtual databases, and used descriptors such as Assisted Reproductive Techniques, Fresh embryo transfer, Frozen-thawed embryo transfer, Freeze-all embryo transfer. Quality randomized controlled trials comparing transfer techniques were included. The variables of interest analyzed were the rates of pregnancy, clinical pregnancy, ongoing pregnancy and abortion, with each study containing at least one of these. Results: Five studies were selected. For rates of clinical pregnancy and ongoing pregnancy, there was greater success for the freeze-all strategy. There was no statistical difference for the rate of chemical pregnancy. The abortion rate had in the transfer of frozen a protective factor. Conclusions: Among the analyzed variables, it was indicated that the freeze-all strategy stands out. This leads us to a direction that the freezing techniques have already reached a level where we can, fearless, wait for a next cycle, that is more physiological and that the technique is better tolerated by the female organism.
\end{abstract}

Keywords: assisted reproductive techniques; embryo transfer; fertilization in vitro.

\section{Introduction}

In assisted human reproduction techniques, embryo transfer can be split into three main categories: fresh embryo transfer; fresh embryo transfer with freezing of high quality supernumerary embryos and freezing all embryos for transfer in a later cycle. Although fresh embryo transfer is the most common procedure, it has a low success rate. ${ }^{1}$

The freeze-all strategy has emerged as an alternative to fresh embryo transfer to improve IVF results. Studies have shown that this technique provides a more physiological environment for the embryo in which the entire cohort of embryos is cryopreserved to be transferred in a subsequent, natural or hormonally programmed cycle for endometrial preparation. $^{2}$

This technique had its clinical value increased according to the significant technological advance in the ability to freeze cleavage and blastocyst stage embryos. Thus, it decreases the reliance on fresh embryo transfer, offering more time to the assisted reproduction cycle and avoiding the late form of the main cause of morbidity and the only cause of mortality of IVF - Ovarian Hyperstimulation Syndrome (OHSS). ${ }^{1}$

Financial support: None.

Conflicts of interest: The authors declare no conflicts of interest.

Submitted: April 19, 2018.

Accepted: May 25, 2018.

The study was carried out at Pontifícia Universidade Católica de Goiás, Goiânia, Goiás, Brazil

Copyright Carmo et al. This is an Open Access article distributed under the terms of the Creative Commons Attribution License, which permits unrestricted use, distribution, and reproduction in any medium, provided the original work is properly cited. 
The use of gonadotrophins as a controlled ovarian stimulation is closely related to the number of antral follicles in order to provide an adequate number of oocytes and avoid OHSS. For each patient, by evaluating the antral follicle count by ultrasound, it is possible to evaluate the ideal dose of gonadotrophin to be used. This is an attempt to reduce the morbimortality of the fresh embryo transfer technique. This procedure needs more evaluation and discussion mainly due to the poor responders and the follicle stimulating hormone (FSH) receptors polymorphisms. ${ }^{3}$

It is important to have a better comparative analysis of both embryo transfer protocols so assisted reproduction services could promote higher success rates and greater safety for patients and embryos.

\section{Methods}

This is a systematic review with meta-analysis. We searched and used the virtual databases Pubmed, EMBASE and Cochrane Central Register of Controlled Trials. The descriptors used to construct the article were: Assisted Reproductive Techniques, Embryo Transfer, Single Embryo Transfer, Fertilization in Vitro, Ovulation Induction, Fresh embryo transfer, Frozen-thawed embryo transfer, Freeze-all embryo transfer. A question and the search were conducted by two independent and blind researchers. In case of disagreement, a third researcher was questioned at weekly consensus meetings about the quality and inclusion of the articles, after complete reading and discussion. The collected data were analyzed and interpreted in order to prepare a final report. ${ }^{4}$

The main inclusion criterion was to be a randomized clinical trial, comparing fresh embryo transfer and freeze-all technique in IVF / ICSI cycles with controlled ovarian stimulation. The analyzed variables were pregnancy rate, clinical pregnancy rate, ongoing pregnancy and miscarriage rate. Seventy papers were found, with selection of seven randomized clinical trials. One of them was excluded due to the use of an egg donation program and another due to methodological problems.

\section{Results}

This meta-analysis included five studies.

The study by Shapiro et al. ${ }^{5}$ with hyper responders patients, was a randomized clinical trial, with a total of 120 patients and 101 blastocysts transferred. The mean age was 31.4 years for the fresh-ET group and 30.6 years for the FET group, revealing no statistical difference between pregnancy rates (75.0\% in Fresh-ET and $85.7 \%$ in FET), as well as in those of clinical pregnancy, ongoing pregnancy and miscarriage.

Another study by Shapiro et al. ${ }^{6}$ was a randomized clinical trial with 137 normo responders patients and 103 blastocysts transferred, with mean age of 32.9 and 33 years for the Fresh-ET and FET groups, respectively. Pregnancy rates for the FET and FreshET group were 90 and $67.9 \%$. Other rates were statistically significant and favorable to the FET group. Only the miscarriage rate showed no significant difference between these groups.

In a randomized clinical trial from Chen et al. ${ }^{7}$ a total of 1508 patients with mean age of 28.2 and 28.3 years for the Fresh-ET and FET groups respectively were included, with 1474 blastocysts transferred. Pregnancy rate showed a significant difference between the groups, being $52.7 \%$ for the frozen transfer and $48.8 \%$ for the fresh group. The miscarriage rate was $32.7 \%$ for the Fresh-ET group and $22 \%$ for the other group.

The randomized clinical trial by Vuong et al. ${ }^{8}$, with 782 patients, mean age of 32.1 and 31.8 years for the Fresh-ET and FET groups, respectively, evaluated only the pregnancy rate, which was not significantly different between the study groups.

Coates et al. ${ }^{9}$, conducted a randomized clinical trial with 179 patients and 107 blastocysts transferred, mean age of 36.6 years for the Fresh-ET group and 36.7 for FET. They verified a clinical pregnancy rate significantly higher in the freeze-all technique $(80.3 \%$ versus $60.8 \%)$ compared to fresh embryo transfer. Pregnancy, clinical pregnancy, ongoing pregnancy and miscarriage rates were also analyzed in Odds Ratio graphs.

For the pregnancy rate, three trials were included, and frozen embryo transfer was positively associated to IVF success. However, as shown in the Figure 1, there was no statistically significant difference between the groups $(p=0.1470,95 \% \mathrm{Cl})$.

For the clinical pregnancy rate, four studies were included, revealing that the freeze-all technique presented favorable and statistically significant outcomes, compared to the other group $(p=0.0332,95 \% \mathrm{Cl})$, as seen in Figure 2 . Likewise, for ongoing pregnancy rate, four studies were included, and the freeze-all technique was statistically significant different $(p=0.0162,95 \% \mathrm{Cl})$ compared to fresh embryo transfer (Figure 3).

Regarding the miscarriage rate, three studies were included for analysis, and Figure 4 shows that freeze-all technique is has a positive statistical significance compared to fresh embryo transfer $(p=0.0014,95 \% \mathrm{Cl})$. 


\section{Studies}

Shapiro et al., 2011 (1)

$0.516(0.191-1.393)$

Shapiro et al., 2011 (2)

$0.252(0.088-0.722)$

Chen et al., 2016 (3)

Combined

$P$-value $=0.1470$

OR (IC95\%)

$0.516(0.191-1.393)$

$0.914(0.736-1.135)$

$0.561(0.257-1.226)$

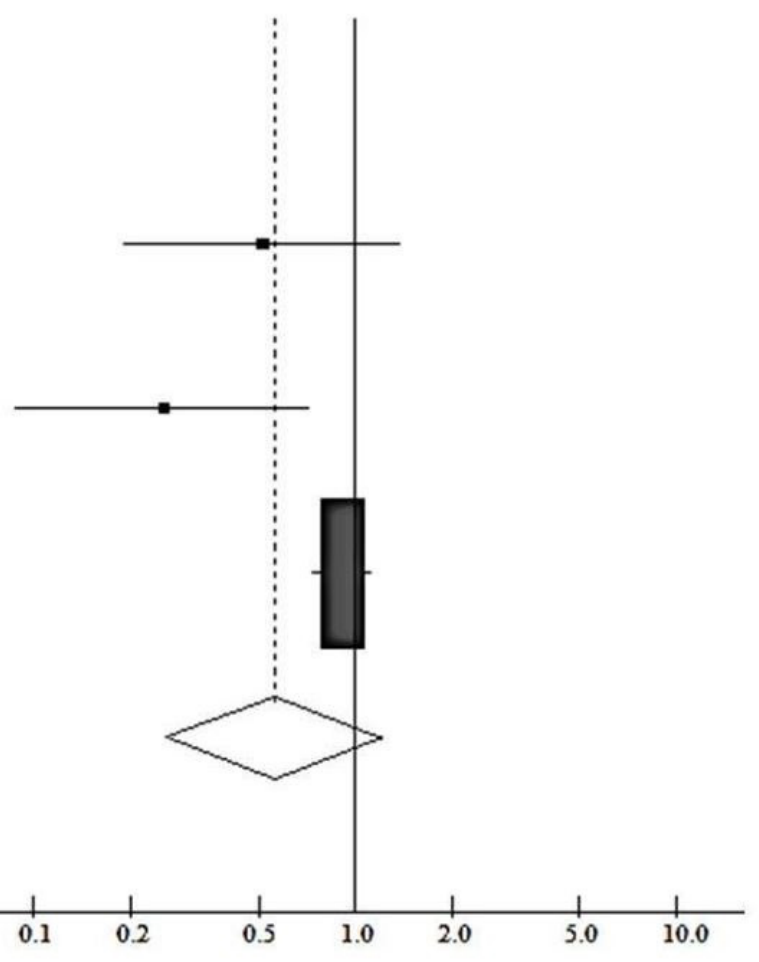

Figure 1. Meta-analysis odds ratio for pregnancy rates.

Studies

Shapiro et al., 2011 (1) $0.496(0.205-1.200)$

Shapiro et al., $2011(2) \quad 0.241$ (0.097-0.599)

Chen et al., $2016(3) \quad 0.880(0.715-1.082)$

Coates et al., 2017 (5)

$0.389(0.166-0.913)$

Combined

$0.492(0.256-0.945)$

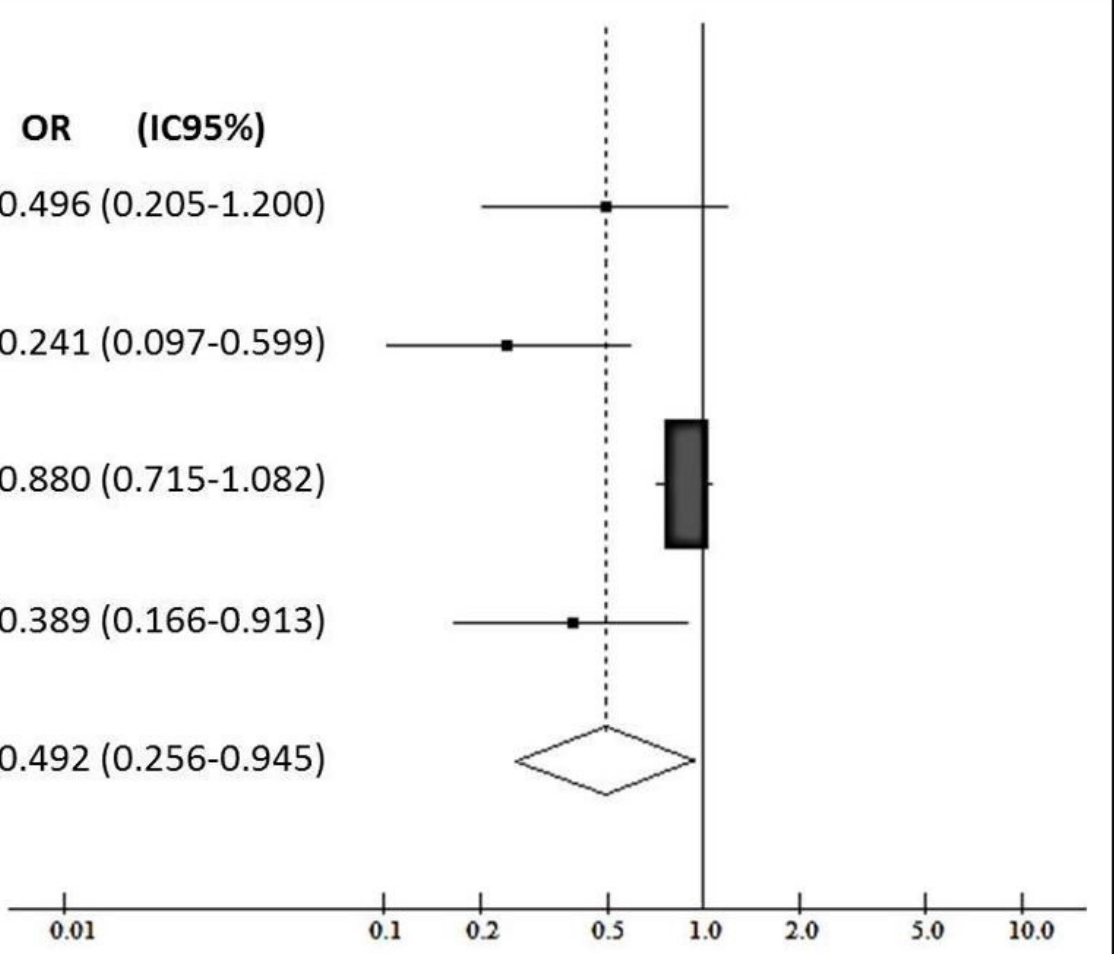

P-value $=0.0332$

Figure 2. Meta-analysis odds ratio for clinical pregnancy rates. 


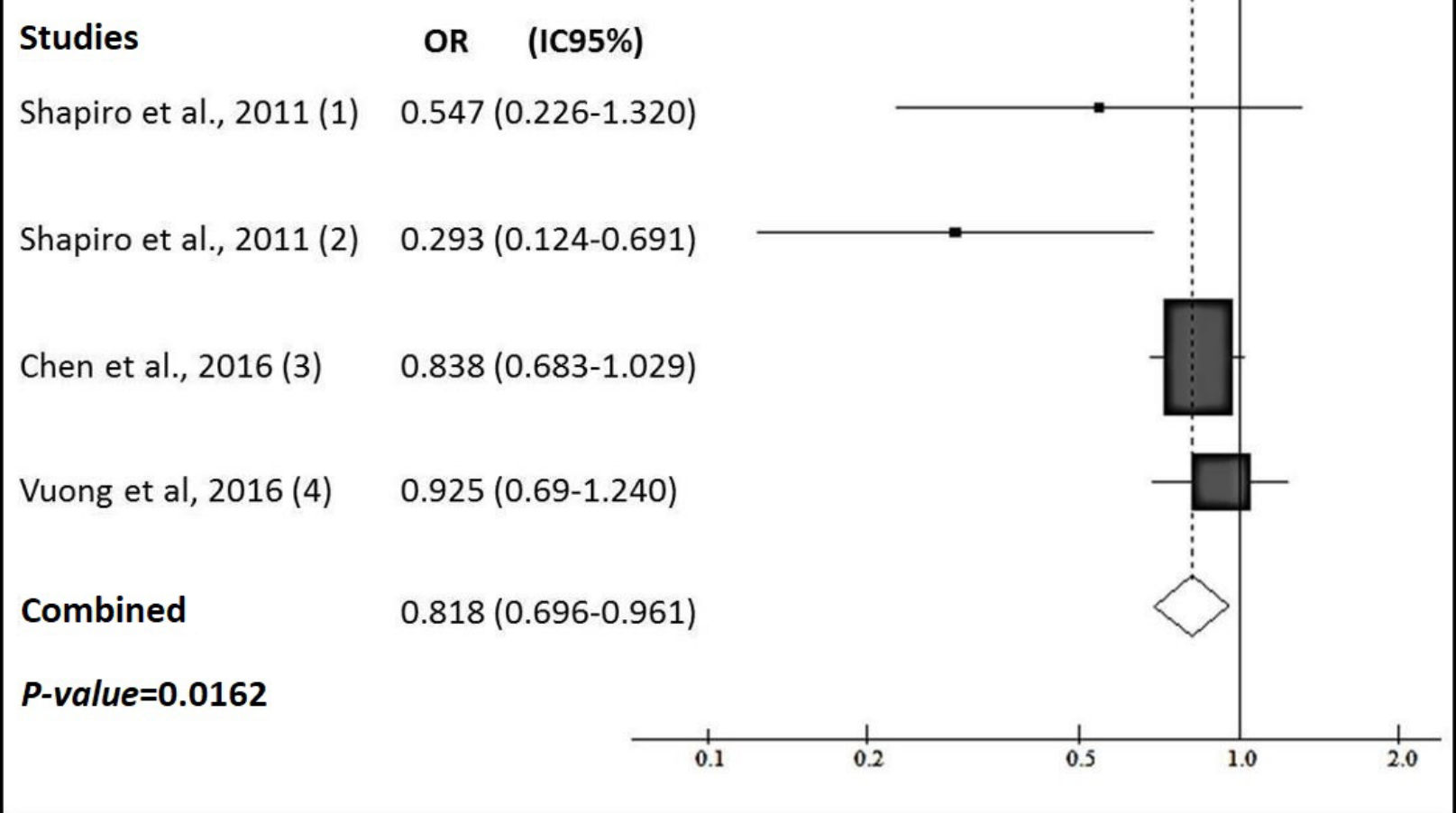

Figure 3. Meta-analysis odds ratio for ongoing pregnancy rates.

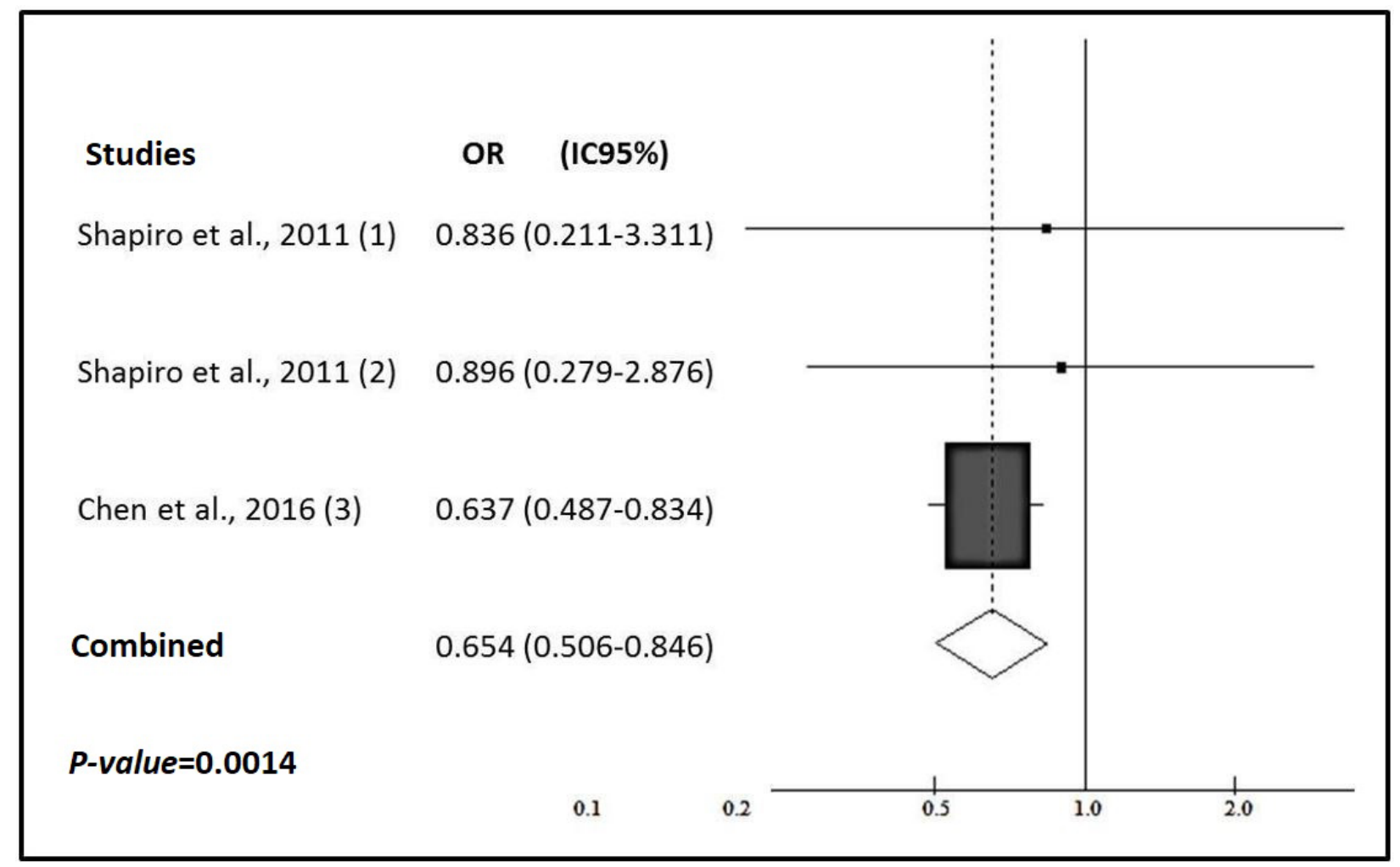

Figure 4. Meta-analysis odds ratio for miscarriage rates. 


\section{Discussion}

In Vitro Fertilization cycles become increasingly studied. The main objective of the techniques evolution is to increase pregnancy rates decreasing potential risks. The fresh embryo transfer technique is still the most widely used due to the historical consolidation, which involves a less complex technical-laboratory apparatus, compared to freeze-all technique. Besides this, it is easier for patient understanding that an embryo produced in the laboratory will be immediately transferred to uterus, since freezing an embryo may be a strange idea for them. ${ }^{1}$

The main risk evolving fresh embryo transfer is the development of Ovarian Hyperstimulation Syndrome, by the use of human chorionic gonadotrophin (hCG) to trigger final oocyte maturation. hCG is a natural hormone, necessary for any pregnancy. It is related to release of pro-implantation factors, and maintenance of the corpus luteum, which produces progesterone. There is, however, a threshold of hCG, which can be reached in a spontaneous pregnancy or, more commonly, in cycles of controlled ovarian stimulation, leading to an exaggerated response. In OHSS there is a great increase of the vascular permeability, being able to culminate even in patient's death. ${ }^{1}$ This threshold is something very subjective, and cannot be predicted in a generalized way. ${ }^{3}$

In freezing-all embryos strategy, there is no need to use hCG, and embryos are transferred in a later cycle into a more physiologic endometrium with only endometrial preparation. Ovarian stimulation is done with alternative techniques, such as the use of GnRH agonists, which allow oocyte retrieval, but is very destructive to the luteal phase. The oocytes are fertilized, embryos are produced and in the later cycle they are transferred according to the endometrial receptivity. 1,7,9

The lack of high quality clinical trials makes it difficult to compare the techniques. Among the analyzed variables, statistical significance was observed in clinical pregnancy, ongoing pregnancy and miscarriage rates favoring freeze-all strategy. This leads us to safe and effective technique, besides being more physiological and better tolerated by the female organism.

Among the selected studies, pregnancy success rates were higher or equal for frozen embryo transfer. It is worth mentioning that in the study by Chen et al. ${ }^{7}$, the miscarriage rate was significantly higher in fresh embryo transfer.

It is important to note that not all selected studies used ovarian stimulation protocols totally hCG-free, or at least did not apply them to all patients. The vitrification idea is to avoid hCG use, but some trials applied low hCG doses in order to retrieve mire oocytes. The only study with exclusive use of the GnRH antagonist protocol was Vuong et al., ${ }^{8}$ who found no significant difference between the success rates of pregnancy between the groups. This study also evaluated the number of women who had moderate to severe hyperstimulation during ovarian stimulation cycles, with no significant difference between the groups. Concerning OHSS, other studies did not present their data.

Thus, it is necessary more systematized studies, using standardized protocols, applied to all patients in each specific group. However, its goal is to extinguish the hyper stimulus risk. There are huge evidences that freeze-all strategy has potential for good results.

\section{References}

1. Evans J, Hannan NJ, Edgell TA, Vollenhoven BJ, Lutjen PJ, Osianlis T, et al. Fresh versus frozen embryo transfer: backing clinical decisions with scientific and clinical evidence. Hum Reprod Update. 2014;20(6):808-21. http://dx.doi.org/10.1093/humupd/ dmu027. PMid:24916455.

2. Braga DP, Setti AS, Figueira RC, Azevedo MC, laconelli Junior A, Turco EGL, et al. Freeze-all, oocyte vitrification, or fresh embryo transfer? Lessons from an egg-sharing donation program. Fertil Steril. 2016;106(3):615-22. http://dx.doi.org/10.1016/j. fertnstert.2016.05.004. PMid:27262501.

3. Castro EC, Borges ALF, Rezende KN, Amaral WN. Antral follicle count in predicting appropriate dose of gonadotropin in in vitro fertilization cycles. Reprod Clim. 2014;29(3):136-42.

4. Castro AA, Guidugli F. Projeto de pesquisa de uma revisão sistemática. In: Castro AA, editor. Planejamento da pesquisa [Internet]. São Paulo: AAC; 2001 [cited 2017 Jul 10]. p. 1-37. Available from: http://www.usinadepesquisa.com/pdf/pesquisar/lv4_15_rsl.PDF

5. Shapiro BS, Daneshmand ST, Garner FC, Aguirre M, Hudson C, Thomas S. Evidence of impaired endometrial receptivity after ovarian stimulation for in vitro fertilization: a prospective randomized trial comparing fresh and frozen-thawed embryo transfers in high responders. Fertil Steril. 2011;96(2):516-8. PMid:21737071.

6. Shapiro BS, Daneshmand ST, Garner FC, Aguirre M, Hudson C, Thomas S. Evidence of impaired endometrial receptivity after ovarian stimulation for in vitro fertilization: a prospective randomized trial comparing fresh and frozen-thawed embryo transfer in normal responders. Fertil Steril. 2011;96(2):344-8. PMid:21737072.

7. Chen ZJ, Shi Y, Sun Y, Zhang B, Liang X, Cao Y, et al. Fresh versus frozen embryos for infertility in the polycystic ovary syndrome. N Engl J Med. 2016;375(6):523-33. http://dx.doi.org/10.1056/NEJMoa1513873. PMid:27509101.

8. Vuong LT, Dang VQ, Ho TM, Huynh BG, Ha DT, Pham TD, et al. Freeze-all versus fresh embryo transfer in IVF/ICSI, a randomised controlled trial. Fertil Steril. 2016;106(3):e376. http://dx.doi.org/10.1016/j.fertnstert.2016.08.006. 
9. Coates A, Kung A, Mounts E, Hesla J, Bankowski B, Barbieri E, et al. Optimal euploid embryo transfer strategy, fresh versus frozen, after preimplantation genetic screening with next generation sequencing: a randomized controlled trial. Fertil Steril. 2017;107(3):723-30. http://dx.doi.org/10.1016/j.fertnstert.2016.12.022. PMid:28139240.

\section{*Correspondence}

Diego Mendes do Carmo

Pontifícia Universidade Católica de Goiás (PUC Goiás),

Avenida T3, 1011, Setor Bueno

CEP 74215-110, Goiânia, GO, Brasil

Tel.: +55 (62) 98303-9992

E-mail: diegomendes_@live.com

\section{Authors information}

DMC - Physician from Pontifícia Universidade Católica de Goiás (PUC Goiás). ECC - PhD in Health Sciences from Universidade Federal de Goiás (UFG); Gynecologist at Humana Medicina Reprodutiva, focus on Human Reproduction; Professor at Pontifícia Universidade Católica de Goiás (PUC Goiás). ARG - Physician from Pontifícia Universidade Católica de Goiás (PUC Goiás); Resident at Santa Casa de Misericórdia de Goiânia. AMTCS - PhD in Cellular and Molecular Biology from Universidade Federal de Goiás (UFG); Professor at Pontifícia Universidade Católica de Goiás (PUC Goiás).

\section{Authors contribution}

DMC was responsible for colecting data and writing the article. ECC reviewed all data at meetings. ARG colected data and wrote the article. AMTCS made statistics and graphs. 